\title{
RELATIVE ARBITRAGE IN VOLATILITY-STABILIZED MARKETS
}

\section{ROBERT FERNHOLZ}

INTECH, One Palmer Square

Princeton, NJ 08542

bob@enhanced.com

\section{IOANNIS KARATZAS}

Departments of Mathematics and Statistics

Columbia University, New York, NY 10027

ik@math. columbia.edu

December 6, 2004

\begin{abstract}
We provide simple, easy-to-test criteria for the existence of relative arbitrage in equity markets. These criteria postulate essentially that the excess growth rate of the market portfolio, a positive quantity that can be estimated or even computed from a given market structure, be "sufficiently large". We show that conditions which satisfy these criteria are manifestly present in the U.S. equity market. We then construct examples of abstract markets in which the criteria hold. These abstract markets allow us to isolate conditions similar to those prevalent in actual markets, and to construct explicit portfolios under these conditions. We study in some detail a specific example of an abstract market which is volatility-stabilized, in that the return from the market portfolio has constant drift and variance rates while the smallest stocks are assigned the largest volatilities. A rather interesting probabilistic structure emerges, in which time changes and the asymptotic theory for planar Brownian motion play crucial roles. The largest stock and the overall market grow at the same, constant rate, though individual stocks fluctuate widely.
\end{abstract}

Key Words and Phrases: Portfolios, relative arbitrage, diversity, volatility-stabilized markets, stochastic differential equations, strict local martingales, time-change, Bessel processes. AMS 2000 Subject Classifications: Primary 60H10, 91B28; secondary 60J55. JEL Subject Classification: G10. 


\section{Introduction and Synopsis}

In the recent monograph Fernholz (2002) and paper Fernholz et al. (2005) we showed that relative arbitrage can exist in equity markets that resemble actual markets. In the cases studied there, arbitrage resulted from market diversity, a condition that prevents the concentration of all the market capital into a single stock. Here we show that diversity is not the proximate cause for the existence of relative arbitrage, but instead this cause appears to lie in a condition related to the variance rates of the stocks in the market. This variance-related condition can pertain even in the absence of diversity.

The excess growth rate of a portfolio of stocks is half the difference between the weighted average of the variances of the stocks in the portfolio, and the portfolio variance (see Fernholz (2002)). Thus, the excess growth rate of the market portfolio provides a measure of the amount of "available volatility" in the market at any given moment. If this available volatility is great enough over a period of time, it can be exploited by certain types of portfolios to outperform the market as shown in Proposition 3.1. We calculate the cumulative excess growth for the U.S equity market, and show that market conditions similar to those of the past century provide sufficient available volatility to permit the existence of arbitrage; see Figure 1 and the discussion following it.

We show that our excess-growth-based criterion is roughly equivalent to market diversity, when the eigenvalues of the market covariance matrix are bounded away from both zero and infinity. However, with unbounded variances, relative arbitrage can exist in the absence of diversity. To study this phenomenon we introduce an example of an abstract market; this is a model that exhibits certain characteristics of a real-life equity market and allows us to focus on those characteristics alone. The abstract market we consider is volatilitystabilized, so the return from the market portfolio has constant drift and variance rates. This stabilization is accomplished by giving the smaller stocks higher volatilities and the bigger stocks smaller volatilities, so that individual stocks "move all over the place" in a sense that we make rigorous (Proposition 4.1), while maintaining overall market stability. The resulting abstract market is not diverse, but nevertheless admits relative arbitrage because the excess growth rate is positive and constant - a feature that makes it rather similar to the real-life U.S. equity market; see Figure 1 and the discussion following it. This example is also of mathematical interest, since the analysis of its behavior involves the asymptotic theory of planar Brownian motion.

In Section 2 we proceed with an introduction to the (rather standard) equity market model that we use, and then present our excess-growth-based criteria for relative arbitrage in Section 3. A generalization of these criteria is also presented, along with results on the minimal time required to "beat the market" by a given amount. Section 4 studies a 
volatility-stabilized market which is not diverse, but which admits relative arbitrage. The technical details of the analysis for this market-model are contained in Section 5, whereas Section 6 studies briefly an extension of the volatility-stabilized model in which the smallest stocks are assigned both big variances and big growth rates.

Some of the proofs of propositions are collected in an Appendix, while several open questions are suggested for further research.

\section{The Model}

Let us consider a model for an equity market with a number $n \geq 2$ of assets, of the form

$$
d X_{i}(t)=X_{i}(t)\left[b_{i}(t) d t+\sum_{\nu=1}^{d} \sigma_{i \nu}(t) d W_{\nu}(t)\right], \quad i=1, \ldots, n .
$$

The quantity $X_{i}(t)$ stands for the value of the $i^{\text {th }}$ asset (stock) at time $t \in[0, \infty)$, and $W_{1}(\cdot), \ldots, W_{d}(\cdot)$ are $d$ independent standard Brownian motions, the "factors" of the model. We shall assume $d \geq n$; namely, that there exist at least as many factors of uncertainty in the market as there are stocks to hedge against them. Another standing assumption is that each stock-issuing company has exactly one share outstanding, so that $X_{i}(t)$ can be interpreted as the capitalization of the $i^{\text {th }}$ company at time $t$.

The vector-valued process $b(\cdot)=\left(b_{1}(\cdot), \ldots, b_{n}(\cdot)\right)^{\prime}$ of rates of return and the $(n \times$ $d)$-matrix-valued process $\sigma(\cdot)=\left\{\sigma_{i \nu}(\cdot)\right\}_{1 \leq i \leq n, 1 \leq \nu \leq d}$ of volatilities are assumed to satisfy the condition

$$
\int_{0}^{T} \sum_{i=1}^{n}\left(\left|b_{i}(t)\right|+\sum_{\nu=1}^{d}\left(\sigma_{i \nu}(t)\right)^{2}\right) d t<\infty, \quad \text { a.s. }
$$

for every $T \in(0, \infty)$. All the processes of this model are defined on a complete probability space $(\Omega, \mathcal{F}, P)$ and are adapted to a given filtration $\mathbf{F}=\{\mathcal{F}(t)\}_{0 \leq t<\infty}$ with $\mathcal{F}(0)=\{\emptyset, \Omega\}$ modulo $P$. This filtration satisfies the "usual conditions" of right-continuity and augmentation by $P$-negligible sets. In particular, $\mathbf{F}$ is allowed to be strictly larger than the filtration generated by the driving Brownian motion $W(\cdot)=\left(W_{1}(\cdot), \ldots, W_{d}(\cdot)\right)^{\prime}$.

The condition (2.2) allows us to write (2.1) in the equivalent form

$$
d\left(\log X_{i}(t)\right)=\gamma_{i}(t) d t+\sum_{\nu=1}^{d} \sigma_{i \nu}(t) d W_{\nu}(t), \quad i=1, \ldots, n
$$

where

$$
\gamma_{i}(t):=b_{i}(t)-\frac{1}{2} a_{i i}(t), \quad a_{i j}(t):=\sum_{\nu=1}^{d} \sigma_{i \nu}(t) \sigma_{j \nu}(t) .
$$


Here $a(\cdot)=\left\{a_{i j}(\cdot)\right\}_{1 \leq i, j \leq n}=\sigma(\cdot) \sigma^{\prime}(\cdot)$ is the variance/covariance matrix-valued process of the model, and we shall refer to $\gamma_{i}(\cdot)$ as the "growth rate" of the $i^{\text {th }}$ asset.

In the context of this model, a portfolio rule is an $\mathbf{F}$-progressively measurable process $\pi(\cdot)=\left(\pi_{1}(\cdot), \ldots, \pi_{n}(\cdot)\right)^{\prime}$ defined on $[0, \infty) \times \Omega$ and with values in the set

$$
\Delta_{+}^{n}:=\left\{\left(x_{1}, \ldots, x_{n}\right) \in \mathbb{R}^{n} \mid x_{1} \geq 0, \ldots, x_{n} \geq 0 \text { and } \sum_{i=1}^{n} x_{i}=1\right\} .
$$

The quantity $\pi_{i}(t)$ is interpreted as the proportion of wealth invested in the $i^{\text {th }}$ stock at time $t$. (There is no hoarding, short-selling of stocks, or borrowing.)

With these assumptions and notation, the value process $Z^{\pi}(\cdot)$ corresponding to this portfolio rule $\pi(\cdot)$ satisfies the equation

$$
\frac{d Z^{\pi}(t)}{Z^{\pi}(t)}=\sum_{i=1}^{n} \pi_{i}(t) \cdot \frac{d X_{i}(t)}{X_{i}(t)}=b^{\pi}(t) d t+\sum_{\nu=1}^{d} \sigma_{\nu}^{\pi}(t) d W_{\nu}(t)
$$

with $Z^{\pi}(0)=z>0$ the initial fortune; here

$$
b^{\pi}(t):=\sum_{i=1}^{n} \pi_{i}(t) b_{i}(t), \quad \sigma_{\nu}^{\pi}(t):=\sum_{i=1}^{n} \pi_{i}(t) \sigma_{i \nu}(t) \quad \text { for } \quad \nu=1, \ldots, d
$$

and

$$
a^{\pi \pi}(t):=\sum_{i=1}^{n} \sum_{j=1}^{n} \pi_{i}(t) a_{i j}(t) \pi_{j}(t)
$$

are, respectively, the rate-of-return coëfficients, the volatility coëfficients and the variance of the portfolio.

By analogy with (2.3), we shall find it convenient to cast the equation (2.5) in the equivalent form

$$
d\left(\log Z^{\pi}(t)\right)=\gamma^{\pi}(t) d t+\sum_{\nu=1}^{d} \sigma_{\nu}^{\pi}(t) d W_{\nu}(t)
$$

with $\gamma^{\pi}(t):=\sum_{i=1}^{n} \pi_{i}(t) \gamma_{i}(t)+\gamma_{*}^{\pi}(t)$ the "growth rate" corresponding to the portfolio rule $\pi(\cdot)$. The quantity

$$
\gamma_{*}^{\pi}(t):=\frac{1}{2}\left(\sum_{i=1}^{n} \pi_{i}(t) a_{i i}(t)-\sum_{i=1}^{n} \sum_{j=1}^{n} \pi_{i}(t) a_{i j}(t) \pi_{j}(t)\right)
$$

is non-negative, and is strictly positive if $\pi_{i}(t)>0$ holds a.s. for all $i=1, \cdots, n$ and $t \geq 0$; see Proposition 1.3.7 in Fernholz (2002). It will be referred to as the excess growth rate of the portfolio $\pi(\cdot)$ and will play an important rôle in what follows.

So will the portfolio rule $\mu(\cdot)=\left(\mu_{1}(\cdot), \cdots, \mu_{1}(\cdot)\right)^{\prime}$ defined by the relative capitalizations

$$
\mu_{i}(t):=\frac{X_{i}(t)}{X_{1}(t)+\cdots+X_{n}(t)}, \quad i=1, \cdots, n
$$


of all the companies in the market. Investing according to this rule amounts to "owning the entire market", in proportion of course to the initial fortune $z>0$. Indeed, it is easily verified from (2.5) and (2.10) that $d Z^{\mu}(t) / Z^{\mu}(t)=d X(t) / X(t)$, where

$$
X(t):=X_{1}(t)+\cdots+X_{n}(t), \quad 0 \leq t<\infty
$$

is the total market capitalization, and this leads to

$$
Z^{\mu}(t)=\frac{z}{x} \cdot\left(X_{1}(t)+\cdots+X_{n}(t)\right), \quad 0 \leq t<\infty \quad \text { with } \quad x:=X_{1}(0)+\cdots+X_{n}(0) .
$$

For this reason, we shall call the rule of (2.10) the market portfolio.

2.1 Definition: Relative Arbitrage. Given any two portfolio rules $\pi(\cdot), \rho(\cdot)$ and a real constant $T>0$, we shall say that $\pi(\cdot)$ is an arbitrage opportunity relative to $\rho(\cdot)$ over the time-horizon $[0, T]$ if there exists a real constant $q \equiv q_{\pi, \rho, T}>0$ such that

$$
\begin{gathered}
P\left(\frac{Z^{\pi}(t)}{Z^{\rho}(t)} \geq q, \quad \forall 0 \leq t \leq T\right)=1, \\
P\left[Z^{\pi}(T) \geq Z^{\rho}(T)\right]=1 \quad \text { and } \quad P\left[Z^{\pi}(T)>Z^{\rho}(T)\right]>0
\end{gathered}
$$

hold, whenever the two portfolio rules start with the same initial fortune $Z^{\pi}(0)=Z^{\rho}(0)=$ $z \in(0, \infty)$.

2.2 Remark. Suppose there exists an $\mathbf{F}$-progressively measurable process $\vartheta:[0, \infty) \times \Omega \rightarrow$ $\mathbb{R}^{d}$ that satisfies the conditions

$$
\sigma(t) \vartheta(t)=b(t), \quad 0 \leq t<\infty
$$

and

$$
\int_{0}^{T}\|\vartheta(t)\|^{2} d t<\infty, \quad \forall 0<T<\infty
$$

almost surely. Such a process is called "relative risk" (or "market price of risk") whenever is exists; see chapter 1 in Karatzas \& Shreve (1998) for discussion. Under these conditions, the exponential process

$$
L(t):=\exp \left\{-\int_{0}^{t} \vartheta^{\prime}(s) d W(s)-\frac{1}{2} \int_{0}^{t}\|\vartheta(s)\|^{2} d s\right\}, \quad 0 \leq t<\infty
$$

is well-defined and is a local martingale and a supermartingale. One checks easily from (2.1), (2.17) that we have

$$
L(t) X_{i}(t)=X_{i}(0) \cdot \exp \left\{-\int_{0}^{t}\left(\eta^{(i)}(s)\right)^{\prime} d W(s)-\frac{1}{2} \int_{0}^{t}\left\|\eta^{(i)}(s)\right\|^{2} d s\right\}
$$


for each $i=1, \cdots, n$, where $\eta^{(i)}:[0, \infty) \times \Omega \rightarrow \mathbb{R}^{d}$ is the vector-valued process defined as $\eta_{\nu}^{(i)}(t):=\vartheta_{\nu}(t)-\sigma_{i \nu}(t)$ for $\nu=1, \cdots, d$ and $0 \leq t<\infty$. Thus, each $L(\cdot) X_{i}(\cdot)$ is also a local martingale and a supermartingale.

More generally, for any portfolio $\pi(\cdot)$ we get

$$
L(t) Z^{\pi}(t)=z \cdot \exp \left\{-\int_{0}^{t}\left(\eta^{\pi}(s)\right)^{\prime} d W(s)-\frac{1}{2} \int_{0}^{t}\left\|\eta^{\pi}(s)\right\|^{2} d s\right\}, \quad 0 \leq t<\infty
$$

from $(2.5),(2.6)$ and $(2.17)$, where $\eta^{\pi}(t):=\vartheta(t)-\sigma^{\prime}(t) \pi(t)$. In particular, for every portfolio rule $\pi(\cdot)$ the process

$$
L(\cdot) Z^{\pi}(\cdot) \quad \text { is a local martingale and a supermartingale . }
$$

2.3 Remark. The excess growth rate of (2.9) can be written as in Lemma 1.3.6, p.20 of Fernholz (2002), in the form

$$
\gamma_{*}^{\pi}(t)=\frac{1}{2} \sum_{i=1}^{n} \pi_{i}(t) \tau_{i i}^{\pi}(t)
$$

where we denote the variances/covariances relative to the portfolio $\pi(\cdot)$, by

$$
\tau_{i j}^{\pi}(t):=\sum_{\nu=1}^{n}\left(\sigma_{i \nu}(t)-\sigma_{\nu}^{\pi}(t)\right)\left(\sigma_{j \nu}(t)-\sigma_{\nu}^{\pi}(t)\right), \quad 1 \leq i, j \leq n .
$$

\section{Sufficient Conditions for Relative Arbitrage}

In terms of the excess growth rate

$$
\gamma_{*}^{\mu}(\cdot)=\frac{1}{2}\left(\sum_{i=1}^{n} \mu_{i}(\cdot) a_{i i}(\cdot)-\sum_{i=1}^{n} \sum_{j=1}^{n} \mu_{i}(\cdot) a_{i j}(\cdot) \mu_{j}(\cdot)\right)=\frac{1}{2} \sum_{i=1}^{n} \mu_{i}(\cdot) \tau_{i i}^{\mu}(\cdot)
$$

of the market portfolio, with the notation of (2.9), (2.10), (2.21) and (2.22), one can formulate easy-to-test sufficient conditions for the existence of relative arbitrage in the model of (2.1), (2.2). We present these sufficient conditions in Propositions 3.1 and 3.4 below.

3.1 Proposition: Suppose there exists a continuous, strictly increasing function $\Gamma$ : $[0, \infty) \rightarrow[0, \infty)$ with $\Gamma(0)=0, \Gamma(\infty)=\infty$ and such that

$$
\Gamma(t) \leq \int_{0}^{t} \gamma_{*}^{\mu}(s) d s<\infty, \quad \forall 0 \leq t<\infty
$$

holds almost surely. Then, with the entropy function $S(x):=-\sum_{j=1}^{n} x_{j} \log x_{j}$, and for any time-horizon $[0, T]$ that satisfies

$$
\Gamma^{-1}(S(\mu(0)))=: T_{*}<T<\infty,
$$


there exists a sufficiently large real number $c>0$ such that the portfolio rule

$$
\pi_{i}(t)=\frac{c \mu_{i}(t)-\mu_{i}(t) \log \mu_{i}(t)}{c-\sum_{j=1}^{n} \mu_{j}(t) \log \mu_{j}(t)}, \quad i=1, \cdots, n
$$

is an arbitrage opportunity relative to the market portfolio; in particular,

$$
P\left[Z^{\pi}(T)>Z^{\mu}(T)\right]=1
$$

Proof: For any given constant $c>0$, let us introduce the 'modified entropy function'

$$
\mathbf{S}_{c}(x):=c+S(x)=c-\sum_{j=1}^{n} x_{j} \log x_{j}, \quad \text { on } \quad \Delta_{++}^{n}=\left\{x \in \Delta_{+}^{n} \mid x_{1}>0, \cdots, x_{n}>0\right\} .
$$

This function is strictly concave and generates the portfolio rule of (3.4) according to the recipe

$$
\pi_{i}(t)=\mu_{i}(t)\left[D_{i} \log \mathbf{S}_{c}(\mu(t))+1-\sum_{j=1}^{n} \mu_{j}(t) D_{j} \log \mathbf{S}_{c}(\mu(t))\right] .
$$

Indeed, with $Z^{\pi}(0)=Z^{\mu}(0)=z>0$ we have the a.s. representation

$$
\log \left(\frac{Z^{\pi}(T)}{Z^{\mu}(T)}\right)=\log \left(\frac{\mathbf{S}_{c}(\mu(T))}{\mathbf{S}_{c}(\mu(0))}\right)+\int_{0}^{T} g(t) d t, \quad 0 \leq T<\infty
$$

(cf. Theorem 3.1.5, pp.46-48 and Example 3.1.2, p.44 in Fernholz (2002)), with the notation of $(2.22)$ and

$$
g(t):=\frac{-1}{2 \mathbf{S}_{c}(\mu(t))} \sum_{i=1}^{n} \sum_{j=1}^{n} D_{i j}^{2} \mathbf{S}_{c}(\mu(t)) \mu_{i}(t) \mu_{j}(t) \tau_{i j}^{\mu}(t),
$$

namely $g(t)=\gamma_{*}^{\mu}(t) / \mathbf{S}_{c}(\mu(t))$ in this case. Now observe that this modified entropy function satisfies the bounds

$$
c<\mathbf{S}_{c}(\cdot) \leq c+\log n, \quad \text { on } \quad \Delta_{++}^{n}
$$

so the first term on the right-hand side of (3.7) dominates $\log \left(\frac{c}{c+S(\mu(0))}\right)$; whereas the last term in (3.7) dominates

$$
\frac{1}{c+\log n} \int_{0}^{T} \gamma_{*}^{\mu}(t) d t \geq \frac{\Gamma(T)}{c+\log n}, \quad \text { a.s. }
$$

thanks to (3.2). It develops that

$$
\log \left(\frac{Z^{\pi}(T)}{Z^{\mu}(T)}\right) \geq \frac{\Gamma(T)}{c+\log n}-\log \left(1+\frac{S(\mu(0))}{c}\right), \quad 0 \leq T<\infty
$$

holds almost surely. In particular, $(2.13)$ is then valid with $\rho(\cdot) \equiv \mu(\cdot)$ and the constant $q=c /(c+S(\mu(0))$. The right-hand side of $(3.9)$ is strictly positive for

$$
T>T_{*}(c):=\Gamma^{-1}\left((c+\log n) \cdot \log \left(1+\frac{S(\mu(0))}{c}\right)\right),
$$


and this leads to (3.5) for every $T>T_{*}(c)$. Since

$$
\lim _{c \rightarrow \infty}\left((c+\log n) \cdot \log \left(1+\frac{S(\mu(0))}{c}\right)\right)=S(\mu(0))
$$

and thus $\lim _{c \rightarrow \infty} T_{*}(c)=T_{*}$ in the notation of (3.3), we see that for every $T>T_{*}$ one can select $c>0$ sufficiently large, so that $T>T_{*}(c)$ and (3.5) hold.

The excess growth rate $\gamma_{*}^{\mu}(t)$ of the market portfolio in (3.1) measures, at any given moment $t$, the amount of "available volatility", namely, the relative variation of the various stocks: this quantity is half the market-weighted average of the intrinsic volatilities $\tau_{i i}^{\mu}(t)$ of the various stocks relative to the market. In such terms, Proposition 3.1 states that if the cumulative available volatility over a period of time is great enough, this volatility can be exploited by a particular functionally generated portfolio, allowing this portfolio to outperform the market.

3.2 Remark: Suppose that the volatilities of the model in (2.1), (2.2) satisfy the strong non-degeneracy condition

$$
\xi^{\prime} \sigma(t) \sigma^{\prime}(t) \xi \geq \varepsilon\|\xi\|^{2}, \quad \forall t \in[0, \infty) \text { and } \xi \in \mathbb{R}^{n}
$$

almost surely, for some real constant $\varepsilon>0$; then the weak diversity condition

$$
P\left[\int_{0}^{T}\left(\max _{1 \leq i \leq n} \mu_{i}(t)\right) d t \leq(1-\delta) T, \quad \forall T \in(0, \infty)\right]=1,
$$

for some $\delta \in(0,1)$, leads to the requirement of $(3.2)$ with $\Gamma(t)=\gamma_{*} t$ and $\gamma_{*}=(\varepsilon \delta) / 2$, and thus also to arbitrages relative to the market portfolio (though not necessarily of the form (3.4)).

Conversely, when we have the a.s. upper bound

$$
\xi^{\prime} \sigma(t) \sigma^{\prime}(t) \xi \leq M\|\xi\|^{2}, \quad \forall t \in[0, \infty) \quad \text { and } \quad \xi \in \mathbb{R}^{n}
$$

for some real constant $M>0$, then (3.2) with $\Gamma(t)=\gamma_{*} t$ and $0<\gamma_{*}<M$ leads to the weak diversity condition of (3.11) for $\delta=\gamma_{*} / M$.

For these facts, see the inequalities (5.12) in Fernholz et al. (2005).

3.3 Remark: From (3.9) we get the a.s. comparison

$$
Z^{\mu}(T) \leq Z^{\pi}(T) \cdot\left(1+\frac{S(\mu(0))}{c}\right) \exp \left\{-\frac{\Gamma(T)}{c+\log n}\right\}, \quad \forall T>T_{*} .
$$


Assuming the existence of a "relative risk" process $\vartheta(\cdot)$ with the properties of (2.15) and (2.16), we see that this inequality, in conjunction with the consequence $E\left[L(T) Z^{\pi}(T)\right] \leq$ $Z^{\pi}(0)=z$ of $(2.20)$, leads to the comparison of expectations

$$
E\left[L(T) Z^{\mu}(T)\right] \leq z\left(1+\frac{S(\mu(0))}{c}\right) \cdot \exp \left\{-\frac{\Gamma(T)}{c+\log n}\right\}, \quad \forall T>T_{*} .
$$

But now, recalling (2.18) and (2.12), we remark that

$$
L(\cdot) X_{1}(\cdot), \cdots, L(\cdot) X_{n}(\cdot) \quad \text { and their sum } \quad L(\cdot) X(\cdot) \equiv \frac{X(0)}{Z^{\mu}(0)} L(\cdot) Z^{\mu}(\cdot)
$$

are all local martingales and supermartingales, for which (3.13) gives

$$
\lim _{T \rightarrow \infty} \downarrow E\left[L(T) X_{1}(T)\right]=\cdots=\lim _{T \rightarrow \infty} \downarrow E\left[L(T) X_{n}(T)\right]=\lim _{T \rightarrow \infty} \downarrow E[L(T) X(T)]=0,
$$

since $\Gamma(\infty)=\infty$. In particular, all the local martingales of (3.14) are strict; and in conjunction with the supermartingale convergence theorem and Fatou's lemma, we deduce

$$
\left(L X_{1}\right)(\infty)=\cdots=\left(L X_{n}\right)(\infty)=(L X)(\infty)=0, \quad \text { a.s. }
$$

3.4 Proposition: Suppose that, in addition to (3.2), (2.2), (2.15) and (2.16), the model of (2.1) satisfies the following property: for every $T>0$ there exists a real constant $K_{T}>0$ such that

$$
\int_{0}^{T} \mu^{\prime}(t) a(t) \mu(t) d t \equiv \int_{0}^{T} \sum_{\nu=1}^{d}\left(\sum_{i=1}^{n} \mu_{i}(t) \sigma_{i \nu}(t)\right)^{2} d t \leq K_{T}
$$

holds a.s. Then the exponential local martingale $L(\cdot)$ of (2.17) is also strict.

Proof: Suppose that the stated conditions are all satisfied, and assume for a moment that $L(\cdot)$ is a martingale. Then for any given $T \in(0, \infty)$ the process

$$
\widetilde{W}(t)=W(t)+\int_{0}^{t} \vartheta(s) d s, \quad 0 \leq t \leq T
$$

is Brownian motion under the equivalent probability measure $\widetilde{P}(A)=\int_{A} L(T) d P$ on $\mathcal{F}(T)$, by the Girsanov theorem (e.g., Karatzas \& Shreve (1991), p.191). And in terms of this process we can write the equation of $(2.5)$ as

$$
d Z^{\pi}(t)=Z^{\pi}(t) \cdot \sum_{\nu=1}^{d}\left(\sum_{i=1}^{n} \pi_{i}(t) \sigma_{i \nu}(t)\right) d \widetilde{W}_{\nu}(t)
$$

for any portfolio rule $\pi(\cdot)$. Now read the equation (3.17) for the market portfolio $\pi(\cdot) \equiv$ $\mu(\cdot)$; the condition (3.16) implies that $Z^{\mu}(\cdot)$ is a $\widetilde{P}$-martingale, or equivalently that $L(\cdot) Z^{\mu}(\cdot) \equiv\left(Z^{\mu}(0) / X(0)\right) L(\cdot) X(\cdot)$ is a $P$-martingale, contradicting (3.15). 
Thus, the local martingale $L(\cdot)$ is strict.

$\diamond$

3.5 Remark: Modifying slightly the proof of Proposition 3.1 one can check that (3.5) holds for some given real constant $T>0$, if there exists a number $\zeta>0$ such that

$$
S(\mu(0))+\zeta \leq \int_{0}^{T} \gamma_{*}^{\mu}(t) d t<\infty \quad \text { holds a.s. }
$$

Figure 1: Cumulative excess growth $\int_{0}^{*} \gamma_{*}^{\mu}(t) d t$ for the U.S. market during $1926-1999$

In Figure 1 we plot the cumulative excess growth, namely, the function $\int_{0}^{*} \gamma_{*}^{\mu}(t) d t$, for the U.S. equity market over most of the twentieth century. The data used for this chart come from the monthly stock database of the Center for Research in Securities Prices (CRSP) at the University of Chicago. The market we construct consists of the stocks traded on the New York Stock Exchange (NYSE), the American Stock Exchange (AMEX) and the NASDAQ Stock Market, after the removal of all REITs, all closed-end funds, and those ADRs not included in the S\&P 500 Index. Until 1962, the CRSP data included only NYSE stocks. The AMEX stocks were included after July 1962, and the NASDAQ stocks were included at the beginning of 1973 . The number of stocks in this market varies from a few hundred in 1926 to about 7500 in 1999.

We note that the cumulative growth $\int_{0}^{\cdot} \gamma_{*}^{\mu}(t) d t$ can be computed in terms of observable quantities such as $\mu(\cdot), Z^{\mu}(\cdot)$ and $Z^{\pi}(\cdot)$, where $\mu(\cdot)$ is the market portfolio and $\pi(\cdot)$ the portfolio rule of (3.4), without any need to estimate the volatility structure $\sigma_{i \nu}(\cdot)$ which is 
not directly observable. This can be done thanks to the formula (3.7), written as

$$
\int_{0}^{T} \gamma_{*}^{\mu}(t) d t=\int_{0}^{T} \mathbf{S}_{c}(\mu(t)) \cdot d\left(\log \left(\frac{Z^{\pi}(t)}{Z^{\mu}(t)} \frac{\mathbf{S}_{c}(\mu(0))}{\mathbf{S}_{c}(\mu(t))}\right)\right), \quad 0 \leq T<\infty .
$$

Figure 1 suggests that the behavior of the U.S. market over most of the twentieth century has exhibited a strictly increasing cumulative excess growth. If the U.S. market continues to behave in a manner that guarantees a positive lower bound on its excess growth rate, then the cumulative excess growth will eventually exceed any finite bound, and in particular the bound of (3.18). Of course, actual markets do not satisfy all the criteria we have imposed here; for instance, stocks enter and leave real markets, so the number of stocks is not constant. Nevertheless, the growth of $\int_{0}^{*} \gamma_{*}^{\mu}(t) d t$ in Figure 1 indicates that the behavior of the U.S. market has been consistent with the existence of relative arbitrage.

\subsection{The Shortest Time to "Beat the Market" by a Given Amount}

What is the shortest time interval $[0, T]$ on which the return $Z^{\varphi}(\cdot)$ from some trading strategy $\varphi(\cdot)$ can exceed the return $Z^{\mu}(\cdot)$ from the market portfolio by a given amount?

To pose this question in a precise manner let us assume that the conditions $(2.15)$ and (2.16) hold, and define a trading strategy $\varphi(t)=\left(\varphi_{1}(t), \cdots, \varphi_{n}(t)\right)^{\prime}$ as a progressively measurable process that satisfies $\int_{0}^{T}\left[\varphi^{\prime}(t) a(t) \varphi(t)+\left|\varphi^{\prime}(t) b(t)\right|\right] d t<\infty$ a.s. for every $T \in(0, \infty)$. Then

$$
\begin{aligned}
Z^{z, \varphi}(t) & =z+\sum_{i=1}^{n} \int_{0}^{t} \varphi_{i}(s) \cdot \frac{d X_{i}(s)}{X_{i}(s)}=z+\sum_{i=1}^{n} \int_{0}^{t} \varphi_{i}(s)\left(b_{i}(t) d t+\sum_{\nu=1}^{d} \sigma_{i \nu}(t) d W_{\nu}(t)\right) \\
& =z+\int_{0}^{t} \varphi^{\prime}(s) \sigma(s)(d W(s)+\vartheta(s) d s), \quad 0 \leq t<\infty .
\end{aligned}
$$

is the value process that corresponds to such a strategy $\varphi(\cdot)$ and to an initial fortune $z>0$. The interpretation here is that $\varphi_{i}(t)$ represents the dollar amount invested in the $i^{\text {th }}$ stock, and $Z^{z, \varphi}(t)-\sum_{i=1}^{n} \varphi_{i}(t)$ the dollar amount kept in the safe, at time $t$. Each of these quantities can take negative values: selling stock short is allowed in this new context, as is borrowing (rather than depositing) money, at zero rate of interest. We require only that the value process satisfy $P\left(Z^{z, \varphi}(t) \geq 0, \forall 0 \leq t<\infty\right)=1$, and denote by $\Phi(z)$ the class of trading strategies with this property. Clearly, this class contains the portfolios of Section 2.

For a given number $h>0$, we are interested in characterizing the smallest length of time

$$
T_{h}:=\inf \left\{T>0 \mid \exists \varphi(\cdot) \in \Phi(1) \text { s.t. } \log \left(Z^{1, \varphi}(T) / Z^{1, \mu}(T)\right) \geq h \text {, a.s. }\right\}
$$


required, to guarantee a log-relative-return vis-à-vis the market portfolio which is at least equal to $h>0$. Recall the notation of (2.11) and (2.12).

3.6 Proposition: Assume the conditions $d=n$, (2.2), (2.15), (2.16) and (3.2) for the model of (2.1); assume also that $\mathbf{F}$ is the augmentation of the filtration generated by the driving Brownian motion $W(\cdot)$. Then the quantity of (3.20) is computed as the value at $e^{-h} \in(0,1)$ of the inverse of the decreasing function $f(T):=\frac{1}{x} E(L(T) X(T))$, namely

$$
T_{h}=\inf \left\{T>0 \mid E(L(T) X(T)) \leq x e^{-h}\right\} .
$$

Proof: First, let us observe that the decreasing function $f(\cdot)$ satisfies $f(0)=1, f(\infty)=0$ from (3.15), and that it is right-continuous; this follows rather easily from Fatou's lemma and the a.s. continuity of the supermartingale $L(\cdot) X(\cdot)$. Now denote by $\widehat{T}_{h}$ the right-hand side of (3.21), and consider an arbitrary element $T>0$ of the set in (3.20). By analogy with (2.20), the supermartingale property of $L(\cdot) Z^{1, \varphi}(\cdot)$ gives

$1=Z^{1, \varphi}(0) \geq E\left(L(T) Z^{1, \varphi}(T)\right) \geq e^{h} E\left(L(T) Z^{1, \mu}(T)\right)=\frac{e^{h}}{x} E(L(T) X(T))=e^{h} f(T) ;$

this means that $T$ belongs also to the set of (3.21), so $\widehat{T}_{h} \leq T_{h}$.

To establish the reverse inequality, consider the number

$$
y:=f\left(\widehat{T}_{h}\right)=\frac{1}{x} E\left(L\left(\widehat{T}_{h}\right) X\left(\widehat{T}_{h}\right)\right)
$$

which lies in the interval $\left(0, e^{-h}\right]$ thanks to the right-continuity of $f(\cdot)$. From the theory of section 9 in Fernholz et al. (2004) we know that there exists a trading strategy $\widehat{\varphi}(\cdot) \in \Phi(y)$ which duplicates exactly the contingent claim $Y:=X\left(\widehat{T}_{h}\right) / x$ at time $t=\widehat{T}_{h}$; to wit, $Z^{y, \widehat{\varphi}}\left(\widehat{T}_{h}\right)=Y$ a.s. But then we get the string of a.s. comparisons

$$
e^{-h} Z^{1, \widehat{\varphi}}\left(\widehat{T}_{h}\right) \geq y Z^{1, \widehat{\varphi}}\left(\widehat{T}_{h}\right)=Z^{y, \widehat{\varphi}}\left(\widehat{T}_{h}\right)=Y=\frac{1}{x} X\left(\widehat{T}_{h}\right)=Z^{1, \mu}\left(\widehat{T}_{h}\right) ;
$$

these imply that $\widehat{T}_{h}$ belongs to the set of (3.20), so $\widehat{T}_{h} \geq T_{h}$ also holds.

We leave to the reader the verification of the following "dual" to this result.

3.7 Proposition: Under the conditions of Proposition 3.6 and for a given time-horizon $T \in(0, \infty)$, the maximal attainable log-relative-return in excess of the market-portfolio, namely

$$
H(T):=\sup \left\{h>0 \mid \exists \varphi(\cdot) \in \Phi(1) \text { s.t. } \log \left(Z^{1, \varphi}(T) / Z^{1, \mu}(T)\right) \geq h \text {, a.s. }\right\},
$$

is given as

$$
H(T)=\log \left(\frac{1}{f(T)}\right)=\log \left(\frac{x}{E(L(T) X(T))}\right) .
$$




\subsection{A Generalization of the Excess Growth Rate}

Let us close this section by mentioning the following generalization of Remark 3.5, which is of considerable independent interest: For any portfolio rule $\pi(\cdot)$ and any number $p \in[0,1]$, we introduce the quantity

$$
\gamma_{*}^{\pi, p}(t):=\frac{1}{2} \sum_{i=1}^{n}\left(\pi_{i}(t)\right)^{p} \tau_{i i}^{\pi}(t)
$$

This generalizes the excess growth rate of $(2.9)$, in the sense that $\gamma_{*}^{\pi, 1}(\cdot) \equiv \gamma_{*}^{\pi}(\cdot)$ holds thanks to (2.21).

3.8 Proposition: Suppose that for some numbers $p \in(0,1), T \in(0, \infty)$ and $\zeta \in(0, \infty)$ we have the condition

$$
\frac{n^{1-p}}{p} \log n+\zeta \leq \int_{0}^{T} \gamma_{*}^{\mu, p}(t) d t<\infty, \quad \text { a.s. }
$$

for the market portfolio, with the notation of (2.10), (3.24). Then the portfolio rule

$$
\pi_{i}(t):=\frac{p\left(\mu_{i}(t)\right)^{p}}{\sum_{j=1}^{n}\left(\mu_{j}(t)\right)^{p}}+(1-p) \mu_{i}(t), \quad i=1, \cdots, n
$$

is an arbitrage opportunity relative to the market portfolio $\mu(\cdot)$, and (3.5) holds.

Proof: Let us read (3.7) and (3.8), (3.6) for the function $\mathbf{S}(x)=\sum_{i=1}^{n} x_{i}^{p}, x \in \Delta_{++}^{n}$, which satisfies $1<\mathbf{S}(\cdot) \leq n^{1-p}$. We deduce from (3.6) that the portfolio rule $\pi(\cdot)$ of (3.26) is generated by this function, and that $g(\cdot)=p(1-p) \gamma_{*}^{\mu, p}(\cdot) / \mathbf{S}(\cdot)$. In particular, we have the a.s. relations

$$
\begin{aligned}
\log \left(\frac{Z^{\pi}(T)}{Z^{\mu}(T)}\right) & =\log \left(\frac{\mathbf{S}(\mu(T))}{\mathbf{S}(\mu(0))}\right)+p(1-p) \int_{0}^{T} \frac{\gamma_{*}^{\mu, p}(t)}{\mathbf{S}(t)} d t \\
& \geq-(1-p) \log n+\frac{p(1-p)}{n^{1-p}} \int_{0}^{T} \gamma_{*}^{\mu, p}(t) d t \geq \frac{p(1-p)}{n^{1-p}} \zeta>0
\end{aligned}
$$

thanks to (3.25), and the claim follows.

We note that (3.25) with $p=1$ implies (3.18), since $S(\mu(0)) \leq \log n$.

3.9 Remark: It should also be noted that a positive lower bound on $\left.\gamma_{*}^{\mu, p}(\cdot)\right|_{p=0}$ is not enough to guarantee the existence of relative arbitrage. Indeed, the model

$$
d\left(\log X_{i}(t)\right)=d W_{i}(t), \quad i=1, \cdots, n
$$


with $\gamma_{i}(\cdot) \equiv 0$ and $\sigma_{i j}(\cdot) \equiv \delta_{i j}(\cdot)$ admits a unique equivalent martingale measure on each time-horizon of finite length, and has bounded variances, so is free of relative arbitrage opportunities. However, $\tau_{i i}^{\mu}(t)=1-2 \mu_{i}(t)+\sum_{j=1}^{n} \mu_{j}^{2}(t) \geq 1-2 \mu_{i}(t)+(1 / n)$, so $\left.2 \gamma_{*}^{\mu, p}(t)\right|_{p=0}=\sum_{i=1}^{n} \tau_{i i}^{\mu}(t) \geq n-1$, a positive constant.

On the other hand, the excess growth rate of the market $\gamma_{*}^{\mu}(t)=\frac{1}{2} \sum_{i=1}^{n} \mu_{i}(t) \tau_{i i}^{\mu}(t)$ $=\frac{1}{2}\left(1-\sum_{i=1}^{n} \mu_{i}^{2}(t)\right)$ is not bounded away from zero; and the condition (3.2) fails in this case.

Open Question: Can the constant $S(\mu(0))$ (respectively, $\left(n^{1-p} \log n\right) / p$ ) be removed from (3.18) (respectively, from (3.25))? In other words, is it the case that (3.5) holds for some given real constant $T>0$, if there exist numbers $\zeta>0$ and $p \in(0,1]$ such that

$$
\zeta \leq \int_{0}^{T} \gamma_{*}^{\mu, p}(t) d t<\infty
$$

holds almost surely?

\section{An Example of Stabilization by Volatility}

In this section and the next we shall study an abstract market model that exhibits, in a parsimonious manner, some of the real-life equity market features we have mentioned: largest volatilities for the smallest stocks, smallest volatilities for the largest stocks, constant drift and variance rates for the market as a whole ('stabilization by volatility'), and a constant positive excess growth rate. Thus, without being diverse, this model contains relative arbitrage opportunities. The model can be generalized in may ways without compromising the main message; one such generalization is offered in section 6 .

In its simplest possible form, this model is of the type (2.3) with

$$
d\left(\log X_{i}(t)\right)=\frac{1}{\sqrt{\mu_{i}(t)}} d W_{i}(t), \quad i=1, \cdots, n, \quad d=n .
$$

In other words, we are selecting equal "growth rates" $\gamma_{i}(\cdot) \equiv 0$ for all the individual stocks; volatilities $\sigma_{i \nu}(\cdot)=\delta_{i \nu} / \sqrt{\mu_{i}(\cdot)}$ in $(2.3)$ which are very high for the smallest stocks and very low for the largest; and covariances of the form $a_{i j}(\cdot)=\delta_{i j} / \mu_{i}(\cdot)$ in $(2.4)$. Similarly, we have

$$
b_{i}(\cdot)=\frac{1}{2 \mu_{i}(\cdot)}, \quad \vartheta_{\nu}(\cdot)=\frac{1}{2 \sqrt{\mu_{\nu}(\cdot)}}
$$

in $(2.1)$ and in (2.15), respectively. Written in the form

$$
d X_{i}(t)=X_{i}(t)\left(\frac{d t}{2 \mu_{i}(t)}+\frac{d W_{i}(t)}{\sqrt{\mu_{i}(t)}}\right), \quad i=1, \cdots, n
$$


of (2.1), the model prescribed by (4.1) amounts to solving in the positive orthant of $\mathbb{R}^{n}$ the following system of stochastic differential equations, for $i=1, \cdots, n$ :

$$
d X_{i}(t)=\frac{1}{2}\left(X_{1}(t)+\cdots+X_{n}(t)\right) d t+\sqrt{X_{i}(t)\left(X_{1}(t)+\cdots+X_{n}(t)\right)} d W_{i}(t) .
$$

A general theory for degenerate stochastic differential equations of this type was developed recently by Bass \& Perkins (2002); see in particular their Theorem 1.2 and Corollary 1.3. This theory shows that the system of equations in (4.2) has a weak solution, which is unique in the sense of the probability law - or equivalently, that the associated martingale problem with infinitesimal generator

$$
(\mathcal{A} f)(x)=\frac{1}{2}\left(x_{1}+\cdots+x_{n}\right) \sum_{i=1}^{n}\left(x_{i} D_{i i}^{2} f(x)+D_{i} f(x)\right), \quad x \in(0, \infty)^{n}
$$

is well-posed. As we shall see in section 5, such a weak solution can be constructed fairly easily in terms of simple time changes, and takes values in $(0, \infty)^{n}$; cf. the representations of (4.15) below. Thus, we can ascertain that the model posited in (4.1) is also well-posed, and as a result the real-valued process $\log X_{i}(\cdot)$ is a local martingale for each $i=1, \cdots, n$ with quadratic variation

$$
T<\int_{0}^{T} \frac{1}{\mu_{i}(t)} d t=\left\langle\log X_{i}\right\rangle(T)<\infty, \quad \text { a.s. }
$$

for every $T \in(0, \infty)$. But

$$
\int_{0}^{T} a_{i i}(t) d t=2 \int_{0}^{T} b_{i}(t) d t=4 \int_{0}^{T}\left(\vartheta_{i}(t)\right)^{2} d t=\int_{0}^{T} \frac{d t}{\mu_{i}(t)},
$$

so the conditions of $(2.2),(2.15),(2.16)$ and (3.10) are all satisfied.

An elementary computation yields the quantities of (2.7)-(2.9) in the form

$$
\gamma^{\mu}(\cdot) \equiv \gamma_{*}^{\mu}(\cdot) \equiv \frac{n-1}{2}=: \gamma_{*}>0 \quad \text { and } \quad a^{\mu \mu}(\cdot) \equiv 1 .
$$

On the other hand, by analogy with (2.6)-(2.9), (4.1) and with the notation of (2.11), (4.5), we see from (4.2) that

$$
d Z^{\mu}(t)=Z^{\mu}(t) \cdot \sum_{i=1}^{n} \mu_{i}(t)\left(d X_{i}(t) / X_{i}(t)\right)=Z^{\mu}(t) \cdot \sum_{i=1}^{n}\left[(1 / 2) d t+\sqrt{\mu_{i}(t)} d W_{i}(t)\right] .
$$

The solution to this equation is given as

$$
\log \left(\frac{Z^{\mu}(t)}{Z^{\mu}(0)}\right)=\gamma_{*} t+B(t), \quad \text { with } \quad B(t):=\sum_{\nu=1}^{n} \int_{0}^{t} \sqrt{\mu_{\nu}(s)} d W_{\nu}(s), \quad 0 \leq t<\infty
$$


a Brownian motion. Indeed, $B(\cdot)$ is clearly a local martingale and its quadratic variation is given by $\langle B\rangle(t)=\int_{0}^{t}\left(\sum_{\nu=1}^{n} \mu_{\nu}(s)\right) d s=t$, so the claim is a consequence of P. Lévy's characterization of the Brownian motion process (e.g. p.157 in Karatzas \& Shreve (1991)). To wit: despite the erratic, widely fluctuating behavior of individual stocks, the overall market performance is remarkably stable.

Discussion: We call this phenomenon stabilization by volatility, as the recipe of (4.1) prescribes big volatility swings for the smallest stocks and smaller volatility swings for the largest stocks, in a way that ends up stabilizing the overall market by producing constant, positive overall growth and variance rates as in (4.5). By contrast, in the so-called "Atlas Model" pioneered by Fernholz (2002) and studied recently in Banner et al. (2004), a similar stabilization for the overall market is achieved by propping up the growth rates of the small stock(s) and keeping those of the large stock(s) minuscule, while each stock is assigned a constant volatility depending on its rank. Here, the quantities $\gamma_{i}(\cdot)$ are equal to zero for all individual stocks. In Section 6 we shall consider briefly a "hybrid" model, which assigns big variances and big growth rates to the smallest stocks.

Let us also observe that the condition (3.2) is satisfied with $\Gamma(t)=\gamma_{*} t$ and $\gamma_{*}=(n-1) / 2$ in this example. According to Proposition 3.1, therefore, the model of (4.1) admits relative arbitrage opportunities, at least on time-horizons $[0, T]$ with

$$
T<T_{*}:=\frac{2 S(\mu(0))}{n-1} \leq \frac{2 \log n}{n-1} .
$$

Contrast this situation to the model of (3.27), where the condition (3.2) is violated, and where relative arbitrage does not exist on any finite time horizon.

Open Question: Note that the upper estimate in (4.7) goes to zero as the number of stocks in the market tends to infinity. Does this indicate that there exist relative arbitrage opportunities on arbitrarily short time horizons in the context of the model (4.1)?

\subsection{Long-Term Behavior of Stocks in (4.1)}

We observe from (4.6) that the total market capitalization $X(\cdot)=X_{1}(\cdot)+\cdots+X_{n}(\cdot)$ of (2.11) is the exponential of a Brownian motion with positive drift $\gamma_{*}=(n-1) / 2$, namely

$$
X(t)=x e^{\gamma_{*} t+B(t)}, \quad 0 \leq t<\infty
$$

recall the notation of (2.12) for the the total market capitalization $x$ at $t=0$. The crude bounds

$$
\max _{1 \leq i \leq n} X_{i}(t)=: X_{(1)}(t) \leq X(t) \leq n X_{(1)}(t)
$$


show that the largest stock and the overall market have the same long-term-growth rate:

$$
\lim _{t \rightarrow \infty}\left(\frac{1}{t} \log X_{(1)}(t)\right)=\lim _{t \rightarrow \infty}\left(\frac{1}{t} \log X(t)\right)=\gamma_{*}, \quad \text { a.s. }
$$

Despite the overall market stability suggested by (4.8) and (4.9), individual stocks "move all over the place", in a sense made precise by the following result.

4.1 Proposition: For every $i=1, \cdots, n$ we have almost surely

$$
\limsup _{t \rightarrow \infty}\left(\frac{1}{t} \log X_{i}(t)\right)=\gamma_{*}, \quad \liminf _{t \rightarrow \infty}\left(\frac{1}{t} \log X_{i}(t)\right)=-\infty .
$$

Proof: Let us apply Itô's rule to the process $\sqrt{X_{i}(\cdot)}$ in conjunction with (4.2), and obtain

$$
\sqrt{X_{i}(t)}=\sqrt{X_{i}(0)}+\int_{0}^{t} \frac{d \Lambda(s)}{2 \sqrt{X_{i}(s)}}+\widehat{W}_{i}(\Lambda(t)), \quad i=1, \cdots, n .
$$

We have introduced here the continuous, strictly increasing time change

$$
\Lambda(t):=\int_{0}^{t}\left(\frac{X(s)}{4}\right) d s=\frac{x}{4} \int_{0}^{t} e^{\gamma_{*} s+B(s)} d s, \quad 0 \leq t<\infty
$$

and the processes

$$
\widehat{W}_{i}(u):=\int_{0}^{\Lambda^{-1}(u)} \sqrt{\Lambda^{\prime}(\xi)} d W_{i}(\xi), \quad 0 \leq u<\infty
$$

for $i=1, \cdots, n$. Note that $\left\langle\widehat{W}_{i}, \widehat{W}_{j}\right\rangle(u)=u \delta_{i j}$ so that, by the P. Lévy characterization once again, the processes $\widehat{W}_{1}(\cdot), \cdots, \widehat{W}_{n}(\cdot)$ are independent Brownian motions.

It follows now that each process $R_{i}(\cdot):=\sqrt{X_{i}\left(\Lambda^{-1}(\cdot)\right)}$ satisfies the stochastic equation

$$
R_{i}(u)=\sqrt{X_{i}(0)}+\int_{0}^{u} \frac{d \xi}{2 R_{i}(\xi)}+\widehat{W}_{i}(u), \quad 0 \leq u<\infty
$$

for the radial part of a planar Brownian motion (or "two-dimensional Bessel") process; see, for instance, Karatzas \& Shreve (1991), p.159. It is checked readily that the squared-Bessel processes $Q_{i}(\cdot):=\left(R_{i}(\cdot)\right)^{2}=X_{i}\left(\Lambda^{-1}(\cdot)\right)$ satisfy the following stochastic equations

$$
d Q_{i}(u)=m d u+2 \sqrt{\left(Q_{i}(u)\right)^{+}} \cdot d \widehat{W}_{i}(u), \quad i=1, \cdots, n
$$

with $m=2$.

According to results of Yamada-Watanabe (ibid., Proposition 2.13 on p.291 and Proposition 3.20 on p.309), each one of these equations admits a pathwise unique, strong solution. 
Thus uniqueness in the sense of the probability law also holds, the squared-Bessel processes $\left(R_{1}(\cdot)\right)^{2}, \cdots,\left(R_{n}(\cdot)\right)^{2}$ are independent, and we obtain the representations

$$
\left(R_{i}(u)\right)^{2}=\left(\rho_{i}^{(1)}+\beta_{i}^{(1)}(u)\right)^{2}+\left(\rho_{i}^{(2)}+\beta_{i}^{(2)}(u)\right)^{2}, \quad 0 \leq u<\infty .
$$

Here $\left(\beta_{i}^{(1)}(\cdot), \beta_{i}^{(2)}(\cdot)\right), i=1, \cdots, n$ are independent standard planar Brownian motions with $\beta_{i}^{(1)}(0)=\beta_{i}^{(2)}(0)=0$ and $\left(\rho_{i}^{(1)}\right)^{2}+\left(\rho_{i}^{(2)}\right)^{2}=X_{i}(0)$. Consequently,

$$
X_{i}(t)=\left(R_{i}(\Lambda(t))\right)^{2}=\left.\left(\left(\rho_{i}^{(1)}+\beta_{i}^{(1)}(u)\right)^{2}+\left(\rho_{i}^{(2)}+\beta_{i}^{(2)}(u)\right)^{2}\right)\right|_{u=\Lambda(t)}, \quad 0 \leq t<\infty
$$

and we obtain

$$
X(t)=X_{1}(t)+\cdots+X_{n}(t)=x e^{\gamma_{*} t+B(t)}=4 \Lambda^{\prime}(t)=(R(\Lambda(t)))^{2}, \quad 0 \leq t<\infty
$$

from (4.8), (4.11) and (4.15), where we have denoted by

$$
R(\cdot):=\sqrt{\left(R_{1}(\cdot)\right)^{2}+\cdots+\left(R_{n}(\cdot)\right)^{2}}=\sqrt{\sum_{i=1}^{n}\left(\left(\rho_{i}^{(1)}+\beta_{i}^{(1)}(\cdot)\right)^{2}+\left(\rho_{i}^{(2)}+\beta_{i}^{(2)}(\cdot)\right)^{2}\right)}
$$

the radial part of the resulting $2 n$-dimensional Brownian motion. In the Appendix we shall verify the property

$$
\lim _{u \rightarrow \infty}\left(\frac{\log R_{i}(u)}{\log u}\right)=\frac{1}{2}, \quad \text { in probability }
$$

so that we have also

$\lim _{t \rightarrow \infty}\left(\frac{1}{t} \log X_{i}(t)\right)=2 \lim _{t \rightarrow \infty}\left(\left.\frac{\log R_{i}(u)}{\log u}\right|_{u=\Lambda(t)} \cdot \frac{\log \Lambda(t)}{t}\right)=\gamma_{*} \quad$ in probability.

The convergence in probability cannot be strengthened to almost-sure convergence in the equations (4.18) or (4.19); indeed, it is also shown in the Appendix that

$$
\limsup _{u \rightarrow \infty}\left(\frac{\log R_{i}(u)}{\log u}\right)=\frac{1}{2} \quad \text { and } \quad \liminf _{u \rightarrow \infty}\left(\frac{\log R_{i}(u)}{\log u}\right)=-\infty
$$

hold almost surely, so that (4.10) follows.

$\diamond$

Comparing (4.19) and (4.10) with (4.9) we see that

$$
\lim _{t \rightarrow \infty}\left(\frac{1}{t} \log \mu_{i}(t)\right)=0 \quad \text { in probability, }
$$


but also that

$$
\limsup _{t \rightarrow \infty}\left(\frac{1}{t} \log \mu_{i}(t)\right)=0, \quad \liminf _{t \rightarrow \infty}\left(\frac{1}{t} \log \mu_{i}(t)\right)=-\infty
$$

hold almost surely. In the terminology of Definition 2.1.1 in Fernholz (2002), this model fails to be coherent in the a.s. sense postulated there - but is "coherent in probability" in the weaker sense of (4.21).

4.2 Remark: Some strict local martingales. For the model of (4.1) we know from Remark 3.1 that the local martingales $L(\cdot) X_{1}(\cdot), \cdots, L(\cdot) X_{n}(\cdot)$ and $L(\cdot) X(\cdot)$ are strict. On the other hand, the exponential local martingale $L(\cdot)$ of $(2.17)$ takes the form

$$
\begin{aligned}
\log \left(\frac{1}{L(T)}\right) & =\frac{1}{2} \sum_{i=1}^{n}\left(\int_{0}^{T} \frac{d W_{i}(t)}{\sqrt{\mu_{i}(t)}}+\frac{1}{4} \int_{0}^{T} \frac{d t}{\mu_{i}(t)}\right) \\
& =\frac{1}{2} \sum_{i=1}^{n}\left(\log \left(\frac{X_{i}(T)}{X_{i}(0)}\right)+\frac{1}{4} \int_{0}^{T} \frac{X_{1}(t)+\cdots+X_{n}(t)}{X_{i}(t)} d t\right)
\end{aligned}
$$

for $0 \leq T<\infty$, or equivalently

$$
L(T)=\sqrt{\frac{X_{1}(0) \cdots X_{n}(0)}{X_{1}(T) \cdots X_{n}(T)}} \exp \left\{-\frac{1}{8} \int_{0}^{T}\left(\sum_{i=1}^{n} X_{i}(t)\right)\left(\sum_{i=1}^{n} \frac{1}{X_{i}(t)}\right) d t\right\} .
$$

Because $\sum_{\nu=1}^{n} \int_{0}^{T}\left(\sum_{i=1}^{n} \mu_{i}(t) \sigma_{i \nu}(t)\right)^{2} d t=\sum_{\nu=1}^{n}\left(\int_{0}^{T} \mu_{\nu}(t) d t\right)=T$, the condition (3.16) is satisfied and the local martingale $L(\cdot)$ is also strict, from Proposition 3.4.

Open Question: With $L(\cdot)$ determined as in (4.22), compute the function $E(L(T) X(T))$ that appears in Propositions 3.6, 3.7 and determine, with the help of (3.21), the shortest time-interval on which it is possible to exceed the return from the market portfolio by a given amount.

4.3 Remark: Lamperti Representations. The last equality in (4.16), namely

$$
x e^{\frac{n-1}{2} t+B(t)}=R^{2}\left(\frac{x}{4} \int_{0}^{t} e^{\frac{n-1}{2} s+B(s)} d s\right), \quad 0 \leq t<\infty,
$$

is equivalent to the Lamperti Representation (e.g. Yor (1992), p.204)

$$
\sqrt{x} e^{(n-1) \theta+\widetilde{B}(\theta)}=R\left(x \int_{0}^{\theta} e^{2[(n-1) s+\widetilde{B}(s)]} d s\right), \quad 0 \leq \theta<\infty
$$

for the exponential of the standard Brownian motion $\widetilde{B}(\cdot):=\frac{1}{2} B(4 \cdot)$ with drift $n-1$, in terms of a time-changed, $2 n$-dimensional Bessel process $R(\cdot)$. 


\subsection{On the distribution of market weights}

The representations of (4.15) and (4.16) offer the opportunity to answer questions about the distribution of the relative market weights $\mu_{i}(\cdot)=X_{i}(\cdot) / X(\cdot)$ in the context of the model (4.1). Here are some results in this direction.

4.4 Proposition: For every $u \in[0, \infty), i=1, \cdots, n$ and $\delta \in(0,1)$ we have

$$
\lim _{u \rightarrow \infty} P\left[\mu_{i}\left(\Lambda^{-1}(u)\right) \leq 1-\delta\right]=1-\delta^{n-1},
$$

where $\Lambda^{-1}(\cdot)$ is the inverse of the continuous, strictly increasing process $\Lambda(\cdot)$ of (4.11).

Proof: From (4.15), (4.16) we have

$$
\mu_{i}\left(\Lambda^{-1}(u)\right)=\left.\frac{X_{i}(t)}{X(t)}\right|_{t=\Lambda^{-1}(u)}=\frac{R_{i}^{2}(u)}{R_{1}^{2}(u)+\cdots+R_{n}^{2}(u)}=\frac{F_{i}(u)}{(n-1)+F_{i}(u)}
$$

for $i=1, \cdots, n$, where the random variable

$$
F_{i}(u):=\frac{R_{i}^{2}(u)}{2} \cdot \frac{2(n-1)}{\sum_{j \neq i} R_{j}^{2}(u)}=(n-1) \cdot \frac{\left(\rho_{i}^{(1)}+\beta_{i}^{(1)}(u)\right)^{2}+\left(\rho_{i}^{(2)}+\beta_{i}^{(2)}(u)\right)^{2}}{\sum_{j \neq i}\left(\left(\rho_{j}^{(1)}+\beta_{j}^{(1)}(u)\right)^{2}+\left(\rho_{j}^{(2)}+\beta_{j}^{(2)}(u)\right)^{2}\right)}
$$

converges in distribution, as $u \rightarrow \infty$, to the quantity

$$
F_{i}:=(n-1) \cdot \frac{Z_{i}}{\sum_{j \neq i} Z_{j}}=\frac{Z_{i} / 2}{\left(\sum_{j \neq i} Z_{j}\right) /(2 n-2)} .
$$

Here the random variables $Z_{1}, \cdots, Z_{n}$ are independent and identically distributed; their common distribution is $\chi_{2}^{2}$ (chi-square with two degrees of freedom), or equivalently, exponential with parameter $\lambda=1 / 2$.

It follows from this representation that, as $u \rightarrow \infty$, the quantity $F_{i}(u)$ has an asymptotic $\digamma_{m, k}$ distribution; the 'degrees of freedom' are $m=2$ for the variable in the numerator and $k=2(n-1)$ for the variable in the denominator. In particular,

$$
\lim _{u \rightarrow \infty} P\left[F_{i}(u) \leq x\right]=\int_{0}^{x}\left(1+\frac{y}{n-1}\right)^{-n} d y=1-\left(1+\frac{x}{n-1}\right)^{1-n}, \quad 0 \leq x<\infty,
$$

and as a result the variables of (4.24) have common asymptotic distribution

$$
\lim _{u \rightarrow \infty} P\left[\mu_{i}\left(\Lambda^{-1}(u)\right) \leq 1-\delta\right]=\lim _{u \rightarrow \infty} P\left[F_{i}(u) \leq(n-1) \frac{1-\delta}{\delta}\right]=1-\delta^{n-1} .
$$

4.5 Remark: On the distribution of the maximum market weight. With the notation introduced in (4.25), we can write the equation (4.24) in the equivalent form

$$
\mu_{i}\left(\Lambda^{-1}(u)\right)=\frac{\left(\rho_{i}^{(1)}+\beta_{i}^{(1)}(u)\right)^{2}+\left(\rho_{i}^{(2)}+\beta_{i}^{(2)}(u)\right)^{2}}{\sum_{j=1}^{n}\left(\left(\rho_{j}^{(1)}+\beta_{j}^{(1)}(u)\right)^{2}+\left(\rho_{j}^{(2)}+\beta_{j}^{(2)}(u)\right)^{2}\right)}
$$


and notice that $\mu_{i}\left(\Lambda^{-1}(u)\right) \longrightarrow Z_{i} /\left(Z_{1}+\cdots+Z_{n}\right)$ in distribution as $u \rightarrow \infty$. From this representation we can obtain in principle the distribution of the maximum market weight along the clock $\Lambda^{-1}(\cdot)$, namely

$$
\begin{array}{r}
\lim _{u \rightarrow \infty} P\left[\max _{1 \leq i \leq n} \mu_{i}\left(\Lambda^{-1}(u)\right) \leq \varrho\right]=\lim _{u \rightarrow \infty} P\left[Z_{i} \leq \varrho\left(Z_{1}+\cdots+Z_{n}\right), \forall i=1, \cdots, n\right] \\
=\int \cdots \int_{\Gamma(\varrho)} \lambda^{n} e^{-\lambda\left(z_{1}+\cdots+z_{n}\right)} d z_{1} \cdots d z_{n}, \quad 1 / n<\varrho<1
\end{array}
$$

where $\Gamma(\varrho):=\left\{\left(z_{1}, \cdots, z_{n}\right) \in(0, \infty)^{n} \mid z_{1} \leq \varrho\left(z_{1}+\cdots+z_{n}\right), \cdots, z_{n} \leq \varrho\left(z_{1}+\cdots+z_{n}\right)\right\}$.

For instance, with $n=2$ the integral is $2 \varrho-1$; with $n=3$ the distribution of $(4.26)$ becomes $(3 \varrho-1)^{2}$ for $1 / 3<\varrho \leq 1 / 2$, and $(3 \varrho-1)^{2}-3(2 \varrho-1)^{2}$ for $1 / 2 \leq \varrho \leq 1$; and so on. In general, the integral of (4.26) can be computed as

$$
\sum_{j=0}^{m} \frac{(-1)^{j} n !}{j !(n-j) !}((n-j) \varrho-1)^{n-1}
$$

where $m$ is the unique integer in $\{0,1, \cdots, n-2\}$ that satisfies

$$
\frac{1}{n-m}<\varrho \leq \frac{1}{n-m-1} .
$$

We are indebted to Adrian Banner for the computation of (4.27).

4.6 Remark: The model of (4.1) is not diverse: there is no number $\delta \in(0,1)$ such that

$$
P\left(\max _{1 \leq i \leq n} \mu_{i}(t)<1-\delta, \quad \forall 0 \leq t<\infty\right)=1 .
$$

This follows rather directly from the computation (4.23) of Proposition 4.3, or from (4.26).

Open Question: For fixed $t \in(0, \infty)$, determine the distributions of $\mu_{i}(t), i=1, \cdots, n$ and of the largest $\mu_{(1)}(t):=\max _{1 \leq i \leq n} \mu_{i}(t)$ and smallest $\mu_{(n)}(t):=\min _{1 \leq i \leq n} \mu_{i}(t)$ market weights.

What can be said about the behavior of the averages $\frac{1}{T} \int_{0}^{T} \mu_{(1)}(t) d t$ and $\frac{1}{T} \int_{0}^{T} \mu_{(n)}(t) d t$, as $T \rightarrow \infty$ ? 


\section{$5 \quad$ Solving the Stochastic Equations of (4.1)}

A weak solution of the system of stochastic equations in (4.1) can be constructed from first principles, in terms of simple time changes; the theory of Bass \& Perkins (2002) then guarantees that this weak solution is unique in the sense of the probability law. We shall outline briefly this construction in the present section.

- Analysis: Suppose that we have constructed already a weak solution, and write $X_{i}(t)=$ $e^{Y_{i}(t)}, 0 \leq t<\infty$ so that $(4.1)$ can be re-cast as

$$
d Y_{i}(t)=\sqrt{\frac{e^{Y_{1}(t)}+\cdots+e^{Y_{n}(t)}}{e^{Y_{i}(t)}}} \cdot d W_{i}(t) \quad \text { with } \quad Y_{i}(0)=\log X_{i}(0),
$$

for $i=1, \cdots n$. In other words, the processes $Y_{1}(\cdot), \cdots, Y_{n}(\cdot)$ are continuous local martingales with quadratic variations

$$
\left\langle Y_{i}\right\rangle(t)=\int_{0}^{t}\left(\frac{e^{Y_{1}(s)}+\cdots+e^{Y_{n}(s)}}{e^{Y_{i}(s)}}\right) d s=: A_{i}(t)
$$

and $\left\langle Y_{i}, Y_{j}\right\rangle(\cdot) \equiv 0$ for $i \neq j$. The functions $t \mapsto A_{i}(t)$ are a.s. continuous and strictly increasing; denoting by $u \mapsto \Gamma_{i}(u)$ their inverses, we know from a theorem of F.B. Knight (e.g. Karatzas \& Shreve (1991), p.179) that the processes

$$
B_{i}(u):=Y_{i}\left(\Gamma_{i}(u)\right), \quad 0 \leq u<\infty
$$

are independent Brownian motions for $i=1, \cdots, n$, and that we have the representations

$$
Y_{i}(t)=B_{i}\left(A_{i}(t)\right), \quad X_{i}(t)=e^{B_{i}\left(A_{i}(t)\right)} \quad \text { for } \quad 0 \leq t<\infty, \quad i=1, \cdots, n .
$$

Substituting back into (5.2) we conclude that the continuous, strictly increasing functions $A_{1}(\cdot), \cdots, A_{n}(\cdot)$ satisfy the system of integral equations for $i=1, \cdots, n$ :

$$
A_{i}(t)=\int_{0}^{t}\left(\frac{e^{B_{1}\left(A_{1}(s)\right)}+\cdots+e^{B_{n}\left(A_{n}(s)\right)}}{e^{B_{i}\left(A_{i}(s)\right)}}\right) d s, \quad 0 \leq t<\infty .
$$

- Solvability of (5.5): Now suppose that we are given $n$ independent Brownian motions $B_{1}(\cdot), \cdots, B_{n}(\cdot)$ with $B_{i}(0)=\log X_{i}(0)$, on the same probability space. We seek $n$ continuous, strictly increasing functions $A_{1}(\cdot), \cdots, A_{n}(\cdot)$ that satisfy (5.5).

Such functions can be found as follows: write (5.5) in the form $e^{B_{i}\left(A_{i}(t)\right)} A_{i}^{\prime}(t)=e^{B_{1}\left(A_{1}(t)\right)}$ $+\cdots+e^{B_{n}\left(A_{n}(t)\right)}$, or equivalently as

$$
Q_{i}\left(A_{i}(t)\right)=\int_{0}^{t}\left(e^{B_{1}\left(A_{1}(s)\right)}+\cdots+e^{B_{n}\left(A_{n}(s)\right)}\right) d s=: A(t)
$$


where

$$
Q_{i}(\xi):=\int_{0}^{\xi} e^{B_{i}(u)} d u, \quad 0 \leq \xi<\infty .
$$

The function $[0, \infty) \ni \xi \mapsto Q_{i}(\xi) \in[0, \infty)$ of $(5.7)$ is continuous and strictly increasing; denoting by $P_{i}(\cdot)$ its inverse and recalling $(5.6)$, we can write $(5.5)$ as

$$
A_{i}(t)=P_{i}(A(t)) \quad \text { and } \quad A(t)=\int_{0}^{t} G(A(s)) d s, \quad 0 \leq t<\infty
$$

for $i=1, \cdots, n$, where

$$
G(u):=\sum_{j=1}^{n} Q_{j}^{\prime}\left(P_{j}(u)\right)=\sum_{j=1}^{n} \frac{1}{P_{j}^{\prime}(u)}, \quad 0 \leq u<\infty .
$$

But the integral equation for $A(\cdot)$ in (5.8) can be solved easily, again by time change. We introduce the continuous and strictly increasing function

$$
C(x):=\int_{0}^{x} \frac{d u}{G(u)}=\int_{0}^{x} \frac{d u}{\sum_{j=1}^{n}\left(1 / P_{j}^{\prime}(u)\right)}, \quad 0 \leq x<\infty
$$

and let $A(\cdot)$ be its inverse; namely, $C(A(t))=t$ for $0 \leq t<\infty$. Then $A^{\prime}(t)=1 / C^{\prime}(A(t))=$ $G(A(t))$, exactly as postulated by the integral equation of (5.8).

With $A(\cdot)$ thus determined, the functions $A_{1}(\cdot), \cdots, A_{n}(\cdot)$ are given as $A_{i}(\cdot)=P_{i}(A(\cdot))$ by the first equation in (5.8), and for $0 \leq t<\infty$ we have

$$
X_{i}(t)=e^{B_{i}\left(P_{i}(A(t))\right)}=Q_{i}^{\prime}\left(P_{i}(A(t))\right)=\frac{1}{P_{i}^{\prime}(A(t))}, \quad X(t)=G(A(t)) .
$$

- Synthesis: Once the continuous, strictly increasing processes $A_{1}(\cdot), \cdots, A_{n}(\cdot)$ have been determined starting from the independent Brownian motions $B_{1}(\cdot), \cdots, B_{n}(\cdot)$ as we just outlined, we define the processes $Y_{1}(\cdot), \cdots, Y_{n}(\cdot)$ via $Y_{i}(\cdot)=B_{i}\left(A_{i}(\cdot)\right)$ as in (5.4). These are continuous local martingales with respect to the augmentation $\mathbf{F}=\{\mathcal{F}(t)\}_{0 \leq t<\infty}$ of their natural filtration $\sigma\left(Y_{i}(s) ; 0 \leq s \leq t, i=1, \cdots, n\right)$, and satisfy $\left\langle Y_{i}, Y_{j}\right\rangle(\cdot)=A_{i}(\cdot) \delta_{i j}$, $Y_{i}(0)=\log X_{i}(0)$.

In terms of these processes we define the continuous $\mathbf{F}$-local martingales

$$
W_{i}(t):=\int_{0}^{t} \sqrt{\frac{e^{Y_{i}(s)}}{e^{Y_{1}(s)}+\cdots+e^{Y_{n}(s)}}} \cdot d Y_{i}(s), \quad 0 \leq t<\infty,
$$

now with $\left\langle W_{i}, W_{j}\right\rangle(t)=\delta_{i j} \int_{0}^{t}\left(e^{Y_{1}(s)}+\cdots+e^{Y_{n}(s)}\right)^{-1} e^{Y_{i}(s)} d A_{i}(s)=\delta_{i j} t$ for $1 \leq i, j \leq n$. In other words, $W_{1}(\cdot), \cdots, W_{n}(\cdot)$ are independent Brownian motions, once again from $\mathrm{P}$. Lévy's characterization. The equations of (5.12) then lead to those of (5.1), that we set out to solve. 
Note that we recover the representation $X(t)=X_{1}(t)+\cdots+X_{n}(t)=x e^{\gamma_{*} t+B(t)}$ of (4.8), where the standard one-dimensional Brownian motion $B(\cdot)$ is now expressed, in accordance with (4.6), (4.8) and (5.12), as

$$
B(t)=\sum_{i=1}^{n} \int_{0}^{t} \mu_{i}(s) d Y_{i}(s)=\sum_{i=1}^{n} \int_{0}^{t} \frac{e^{Y_{i}(s)} d Y_{i}(s)}{e^{Y_{1}(s)}+\cdots+e^{Y_{n}(s)}} .
$$

5.1 Remark: From (5.11), (5.8) and (4.10) we have $A(t)=\int_{0}^{t} G(A(s)) d s=\int_{0}^{t} X(s) d s=$ $4 \Lambda(t)$. Comparing this with (4.15)-(4.17) we make the identifications

$$
\begin{gathered}
\Lambda^{-1}(u)=A^{-1}(4 u)=C(4 u), \quad \text { as well as } \\
\frac{1}{P_{i}^{\prime}(u)}=R_{i}^{2}(u / 4), \quad i=1, \cdots, n \quad \text { and } \quad G(u)=R^{2}(u / 4), \quad 0 \leq u<\infty,
\end{gathered}
$$

in terms of the 2-dimensional Bessel processes $R_{i}(\cdot), i=1, \cdots, n$ and the $2 n$-dimensional Bessel process $R(\cdot)$. Back into (5.10) these identifications give the expressions $C(x)=$ $\int_{0}^{x}(G(u))^{-1} d u=\int_{0}^{x} R^{-2}(u / 4) d u=4 \int_{0}^{x / 4} R^{-2}(s) d s$, which in turn imply

$$
\Lambda^{-1}(u)=4 \int_{0}^{u} \frac{d s}{R^{2}(s)}, \quad 0 \leq u<\infty .
$$

In conjunction with $(4.16)$ and with $\widetilde{B}(\cdot)=B(4 \cdot) / 2$, this identification allows us to express the Lamperti Representation of Remark 4.3 in the following equivalent form (see Yor (1992), p. 203, and Jacobsen \& Yor (2003)):

$$
R(u)=\left.\sqrt{x} e^{(n-1) \theta+\widetilde{B}(\theta)}\right|_{\theta=\int_{0}^{u} R^{-2}(s) d s}, \quad 0 \leq u<\infty .
$$

\section{A Hybrid Model}

We shall discuss briefly in this section a generalization of the model of (4.1) which assigns both big variances and big growth rates to the smallest stocks (whence the appellation "hybrid" at the head of the section) but again in such a manner that the overall market performance is remarkably stable. This model is of the form

$$
d\left(\log X_{i}(t)\right)=\frac{\alpha}{2 \mu_{i}(t)} d t+\frac{1}{\sqrt{\mu_{i}(t)}} d W_{i}(t), \quad i=1, \cdots, n,
$$

or equivalently

$$
d X_{i}(t)=\frac{1+\alpha}{2}\left(X_{1}(t)+\cdots+X_{n}(t)\right) d t+\sqrt{X_{i}(t)\left(X_{1}(t)+\cdots+X_{n}(t)\right)} d W_{i}(t)
$$


as in (4.2), where we shall take $\alpha \geq 0$ and set $m:=2(1+\alpha)$. In the notation of section 2 , we have now

$$
\sigma_{i \nu}(t)=\frac{\delta_{i \nu}}{\sqrt{\mu_{\nu}(t)}}, \quad \vartheta_{\nu}(t)=\frac{1+\alpha}{2 \sqrt{\mu_{\nu}(t)}}, \quad \gamma_{i}(t)=\frac{\alpha}{2 \mu_{i}(t)} .
$$

The model studied in Sections 4 and 5 corresponds to the choice $\alpha=0(m=2)$, so we shall concentrate here on $\alpha>0(m>2)$.

It follows again from the theory of Bass \& Perkins (2002) that the martingale problem with infinitesimal generator

$$
(\mathcal{A} f)(x)=\frac{1}{2}\left(x_{1}+\cdots+x_{n}\right) \sum_{i=1}^{n}\left(x_{i} D_{i i}^{2} f(x)+(1+\alpha) D_{i} f(x)\right), \quad x \in(0, \infty)^{n}
$$

suggested by (6.2), is well-posed; the weak solution takes values in $(0, \infty)^{n}$; and straightforward computations give again constant variance and growth rates for the resulting market, namely

$$
a^{\mu \mu}(t) \equiv 1, \quad \gamma_{*}^{\mu}(t) \equiv \gamma_{*}=(n-1) / 2
$$

and

$$
\gamma^{\mu}(t)=\sum_{i=1}^{n} \mu_{i}(t) \frac{\alpha}{2 \mu_{i}(t)}+\gamma_{*}^{\mu}(t) \equiv \frac{(1+\alpha) n-1}{2}=\frac{m n}{4}-\frac{1}{2}=: \gamma>0 .
$$

In particular, the requirements of Propositions 3.1 and 3.4 are satisfied once again; the model admits arbitrage opportunities relative to the market portfolio; the local martingales $L(\cdot) X_{1}(\cdot), \cdots, L(\cdot) X_{n}(\cdot)$ of $(3.14)$ are all strict, as is the exponential local martingale $L(\cdot)$ of (2.17); and the total market capitalization takes again the form

$$
X(t)=X_{1}(t)+\cdots+X_{n}(t)=x e^{\gamma t+B(t)}, \quad 0 \leq t<\infty
$$

of exponentiated Brownian motion $B(\cdot)$ as in (4.6), with drift $\gamma$ given by (6.4).

By analogy with (4.11)-(4.17), we have

$$
X_{i}(t)=R_{i}^{2}(\Lambda(t)), \quad i=1, \cdots, n \quad \text { and } \quad X(t)=R^{2}(\Lambda(t)), \quad 0 \leq t<\infty
$$

where now $\Lambda(\cdot)$ is given by

$$
\Lambda(t):=\int_{0}^{t}\left(\frac{X(s)}{4}\right) d s=\frac{x}{4} \int_{0}^{t} e^{\gamma s+B(s)} d s, \quad \text { thus } \quad \Lambda^{-1}(u)=4 \int_{0}^{u} \frac{d s}{R^{2}(s)}
$$

as in (5.13). Here $R_{1}(\cdot), \cdots, R_{n}(\cdot)$ are independent Bessel processes with 'dimension' $m=$ $2(1+\alpha)$, namely

$$
d R_{i}(u)=\frac{m-1}{2 R_{i}(u)} d u+d \widehat{W}_{i}(u)
$$


and $\widehat{W}_{1}(\cdot), \cdots, \widehat{W}_{n}(\cdot)$ are independent one-dimensional Brownian motions as in (4.12), (4.13). (Equivalently, $Q_{i}(\cdot)=\left(R_{i}(\cdot)\right)^{2}$ satisfies the equations (4.14).) Thus the process $R(\cdot)=\sqrt{R_{1}^{2}(\cdot)+\cdots+R_{n}^{2}(\cdot)}$ is Bessel in 'dimension' $n m$, namely

$$
d R(u)=\frac{m n-1}{2 R(u)} d u+d \widehat{W}(u)
$$

where $\widehat{W}(\cdot)$ is another one-dimensional Brownian motion.

The growth rate for the entire market and for the largest stock is computed easily:

$$
\lim _{t \rightarrow \infty}\left(\frac{1}{t} \log X(t)\right)=\lim _{t \rightarrow \infty}\left(\frac{1}{t} \log X_{(1)}(t)\right)=\gamma, \quad \text { a.s. }
$$

6.1 Proposition: For the model of (6.1) we have for every $i=1, \cdots, n$, almost surely:

$$
\begin{gathered}
\lim _{t \rightarrow \infty}\left(\frac{1}{t} \log X_{i}(t)\right)=\gamma \\
\lim _{T \rightarrow \infty} \frac{1}{T} \int_{0}^{T} a_{i i}(t) d t=\lim _{T \rightarrow \infty} \frac{1}{T} \int_{0}^{T} \frac{d t}{\mu_{i}(t)}=\frac{2 \gamma}{\alpha}=n+\frac{n-1}{\alpha} .
\end{gathered}
$$

Proof: In order to compute the long-term-growth behavior of an individual stock as in (6.10), let us start by observing that we have now the following strengthening

$$
\lim _{u \rightarrow \infty}\left(\frac{\log R_{i}(u)}{\log u}\right)=\frac{1}{2}, \quad \text { a.s. }
$$

of (4.18). Indeed, an application of Itô's rule to (6.8) gives the dynamics $d\left(\log R_{i}(u)\right)=$ $\alpha R_{i}^{-2}(u) d u+R_{i}^{-1}(u) d \widehat{W}_{i}(u)$, so that

$$
\log R_{i}(u)=\frac{1}{2} \log X_{i}(0)+\left.\left(\alpha \theta+\widehat{B}_{i}(\theta)\right)\right|_{\theta=\int_{0}^{u} R_{i}^{-2}(s) d s} .
$$

Equivalently, we have the Lamperti representation

$$
R_{i}(u)=\left.\sqrt{X_{i}(0)} \cdot e^{\alpha \theta+\widehat{B}_{i}(\theta)}\right|_{\theta=\int_{0}^{u} R_{i}^{-2}(s) d s}, \quad 0 \leq u<\infty
$$

as in $(5.14)$, where $\widehat{B}_{i}(\cdot)$ is yet another Brownian motion. Now it can be checked (e.g. Pitman \& Yor (1986.a), p. 112; Yor (1985); or Cherny (2000), p. 206) that

$$
\lim _{u \rightarrow \infty}\left(\frac{1}{\log u} \int_{0}^{u} \frac{d s}{R_{i}^{2}(s)}\right)=\frac{1}{m-2}=\frac{1}{2 \alpha} \quad \text { holds a.s. }
$$


In conjunction with $(6.13)$ and the strong law of large numbers $\lim _{\xi \rightarrow \infty}\left(\widehat{B}_{i}(\theta) / \theta\right)=0$ a.s., the result (6.14) leads to the claim of (6.12), and hence to

$$
\lim _{t \rightarrow \infty} \frac{1}{t} \log X_{i}(t)=2 \cdot \lim _{t \rightarrow \infty}\left(\left.\frac{\log R_{i}(u)}{\log u}\right|_{u=\Lambda(t)} \cdot \frac{\log \Lambda(t)}{t}\right)=\gamma, \quad \text { a.s. }
$$

On the other hand, we notice from (6.5)-(6.7) that

$$
\int_{0}^{T} a_{i i}(t) d t=\int_{0}^{T} \frac{d t}{\mu_{i}(t)}=\int_{0}^{T} \frac{X(t)}{X_{i}(t)} d t=\int_{0}^{T} \frac{4 \Lambda^{\prime}(t) d t}{\left(R_{i}(\Lambda(t))^{2}\right.}=\int_{0}^{\Lambda(T)} \frac{4 d u}{\left(R_{i}(u)\right)^{2}},
$$

and this gives

$$
\lim _{T \rightarrow \infty} \frac{1}{T} \int_{0}^{T} \frac{d t}{\mu_{i}(t)}=\left.\frac{\log \Lambda(T)}{T} \cdot\left(\frac{4}{\log u} \int_{0}^{u} \frac{d s}{R_{i}^{2}(s)}\right)\right|_{u=\Lambda(T)} \longrightarrow \frac{2 \gamma}{\alpha}
$$

almost surely, as $T \rightarrow \infty$.

In particular, (6.15) and (6.9) show that the hybrid model of (6.1) is coherent, in the sense that for every $i=1, \cdots, n$ we have:

$$
\lim _{t \rightarrow \infty}\left(\frac{1}{t} \log \mu_{i}(t)\right)=0
$$

almost surely.

\section{Appendix}

Starting with the pioneering works of Kallianpur \& Robbins (1953) and Spitzer (1958), a rich theory exists now for the asymptotic behavior of additive functionals associated with the planar Brownian motion process. An excellent survey of this subject was carried out by Pitman \& Yor $(1986,1989)$. In particular, equation (2) of Table 1, p.762 in their (1986) paper leads to the result (4.18). There is, however, a very direct argument for this result, pointed out to us by Marc Yor (2004).

Proof of (4.18): From the scaling property of the planar Brownian motion $\beta(\cdot)=$ $\left(\beta^{1}(\cdot), \beta^{2}(\cdot)\right)^{\prime}$, where $\beta^{1}(\cdot)$ and $\beta^{2}(\cdot)$ are standard one-dimensional Brownian motions, and with $\xi \in \mathbb{R}^{2}$ the initial position, we have

$R(u)=\|\xi+\beta(u)\|=\sqrt{u}\|(\xi+\beta(u)) / \sqrt{u}\|=\sqrt{u} \|(\xi / \sqrt{u})+\beta(1)) \|, \quad$ in distribution.

Therefore,

$$
\left.\log R(u)-\frac{1}{2} \log u=\log \|(\xi / \sqrt{u})+\beta(1)\right) \|, \quad \text { in distribution, }
$$


and this leads to

$$
\left.\lim _{u \rightarrow \infty}\left(\log R(u)-\frac{1}{2} \log u\right)=\log \| \beta(1)\right) \|, \quad \text { in distribution }
$$

(that is to say, as $u \rightarrow \infty$ the distribution of $R^{2}(u) / u$ is approximately chi-square with two degrees of freedom). In particular, we have $\lim _{u \rightarrow \infty}(\log R(u) / \log u)=1 / 2$ in distribution, thus also in probability.

Proof of (4.20): The law of the iterated logarithm for the standard, one-dimensional Brownian motion, leads directly to the corresponding result

$$
\limsup _{u \rightarrow \infty}\left(\frac{R(u)}{\sqrt{2 u \log \log u}}\right)=1, \quad \text { a.s. }
$$

for the radial part of the planar Brownian motion. It follows now from (7.2) that

$$
\limsup _{u \rightarrow \infty}\left(\frac{\log R(u)}{\log u}\right)=\frac{1}{2}
$$

holds almost surely.

In order to deal with the limit inferior, we shall rely on the following zero-one law of Spitzer (1958): For a decreasing function $h(\cdot)$ we have

$$
P\left(R(u) \geq u^{1 / 2} h(u) \text { for all } u>0 \text { sufficiently large }\right)=1 \text { or } 0,
$$

depending on whether the series below converges or diverges:

$$
\sum_{k=1}^{\infty} \frac{1}{k|\log h(k)|}
$$

With $h(u)=u^{-f(u)}$ and $f(u)=\log \log u$, the series of (7.4) becomes $\sum_{k=1}^{\infty} \frac{1}{k \log k \log \log k}$ and diverges. Thus from (7.3) and Spitzer's result we obtain that

$$
(\forall \tau>0)(\exists u>\tau) \quad \text { such that } \log R(u)<\frac{1}{2} \log u-\log u \cdot \log \log u
$$

holds almost surely. Dividing through by $\log u$ and then letting $u \rightarrow \infty$, we get

$$
\liminf _{u \rightarrow \infty}(\log R(u) / \log u)=-\infty
$$

almost surely. 


\section{Acknowledgements}

We are indebted to Adrian Banner and Constantinos Kardaras for several helpful discussions; in particular, the computation of (4.27) is due to Adrian Banner. We are also grateful to Professors Chris Rogers and Marc Yor for their expert advice on the subject of planar Brownian motion, and to Professor Peter Bank for an observation that helped us correct an error in an earlier version of the paper.

\section{References}

Banner, A., Fernholz, R. \& Karatzas, I. (2004) On Atlas models of equity markets. Preprint. Bass, R. \& Perkins, E. (2002) Degenerate stochastic differential equations with Höldercontinuous coëfficients and super-Markov chains. Transactions of the American Mathematical Society 355, 373-405.

Cherny, A.S. (2000) Convergence of some integrals associated with Bessel processes. Theory of Probability and Its Applications 45, 195-209.

Fernholz, E.R. (2002) Stochastic Portfolio Theory. Springer-Verlag, New York.

Fernholz, R., Karatzas, I. \& Kardaras, C. (2005) Diversity and relative arbitrage in equity markets. Finance 85 Stochastics 9, 1-27.

Jacobsen, M. \& Yor, M. (2003) Multi-self-similar Markov processes on $\mathbb{R}_{+}^{n}$ and their Lamperti representations. Probability Theory and Related Fields 126, 1-28.

Kallianpur, G. \& Robbins, H. (1953) Ergodic property of the planar Brownian motion process. Proceedings of the National Academy of Sciences 39, 525-533.

Karatzas, I. \& Shreve, S.E. (1991) Brownian Motion and Stochastic Calculus. Second Edition. Springer-Verlag, New York.

Karatzas, I. \& Shreve, S.E. (1998) Methods of Mathematical Finance. Springer-Verlag, New York.

Pitman, J. \& Yor, M. (1986) Asymptotic laws for planar Brownian motion. Annals of Probability 14, 733-779.

Pitman, J. \& Yor, M. (1986.a) Some divergent integrals of Brownian motion. In "Analysis and Geometric Stochastics" in Honor of G.H. Reuter (D. Kendall, editor); supplement to the Advances in Applied Probability 18, 109-116. 
Pitman, J. \& Yor, M. (1989) Further asymptotic laws for planar Brownian motion. Annals of Probability 17, 965-1011.

Spitzer, F. (1958) Some theorems concerning two-dimensional Brownian motion. Transactions of the American Mathematical Society 87, 187-197.

Yor, M. (1985) Renormalisation et convergence en loi pour les temps locaux du mouvement Brownien dans $\mathbb{R}^{n}$. Lecture Notes in Mathematics 1204, 543-552.

Yor, M. (1992) Sur certaines functionelles exponentielles du mouvement Brownien. Journal of Applied Probability 29, 202-208.

Yor, M. (2004) Personal Communication, 27 May 2004. 